\title{
Camil GUY
}

Anthropologue québécois

(1967)

\section{"Les indiens du Québec : désagrégation culturelle et prolétarisation.”}

\author{
Un document produit en version numérique par Jean-Marie Tremblay, bénévole, \\ professeur de sociologie au Cégep de Chicoutimi \\ Courriel: jean-marie_tremblay@uqac.ca \\ Site web pédagogique : http://www.uqac.ca/jmt-sociologue/ \\ Dans le cadre de: "Les classiques des sciences sociales" \\ Une bibliothèque numérique fondée et dirigée par Jean-Marie Tremblay, \\ professeur de sociologie au Cégep de Chicoutimi \\ Site web: http://classiques.uqac.ca/ \\ Une collection développée en collaboration avec la Bibliothèque \\ Paul-Émile-Boulet de l'Université du Québec à Chicoutimi \\ Site web: http://bibliotheque.uqac.ca/
}




\section{Politique d'utilisation de la bibliothèque des Classiques}

Toute reproduction et rediffusion de nos fichiers est interdite, même avec la mention de leur provenance, sans l'autorisation formelle, écrite, du fondateur des Classiques des sciences sociales, Jean-Marie Tremblay, sociologue.

Les fichiers des Classiques des sciences sociales ne peuvent sans autorisation formelle:

- être hébergés (en fichier ou page web, en totalité ou en partie) sur un serveur autre que celui des Classiques.

- servir de base de travail à un autre fichier modifié ensuite par tout autre moyen (couleur, police, mise en page, extraits, support, etc...),

Les fichiers (.html, .doc, .pdf, .rtf, .jpg, .gif) disponibles sur le site Les Classiques des sciences sociales sont la propriété des Classiques des sciences sociales, un organisme à but non lucratif composé exclusivement de bénévoles.

Ils sont disponibles pour une utilisation intellectuelle et personnelle et, en aucun cas, commerciale. Toute utilisation à des fins commerciales des fichiers sur ce site est strictement interdite et toute rediffusion est également strictement interdite.

L'accès à notre travail est libre et gratuit à tous les utilisateurs. C'est notre mission.

Jean-Marie Tremblay, sociologue

Fondateur et Président-directeur général, LES CLASSIQUES DES SCIENCES SOCIALES. 
Cette édition électronique a été réalisée par Jean-Marie Tremblay, bénévole, professeur de sociologie au Cégep de Chicoutimi à partir de :

\section{Camil Guy}

\section{“Les indiens du Québec : désagrégation culturelle et prolétari- sation.”}

Un article publié dans la revue Parti pris, revue politique et culturelle, vol. 4, nos 9-12, mai-août 1967, pp. 165-181.

Polices de caractères utilisée :

Pour le texte: Times New Roman, 14 points.

Pour les notes de bas de page : Times New Roman, 12 points.

Édition électronique réalisée avec le traitement de textes Microsoft Word 2008 pour Macintosh.

Mise en page sur papier format : LETTRE US, 8.5'” x 11'”.

Édition numérique réalisée le 2 septembre 2012 à Chicoutimi, Ville de Saguenay, Québec. 


\section{Camil GUY}

Anthropologue québécois

"Les indiens du Québec:

désagrégation culturelle et prolétarisation.”

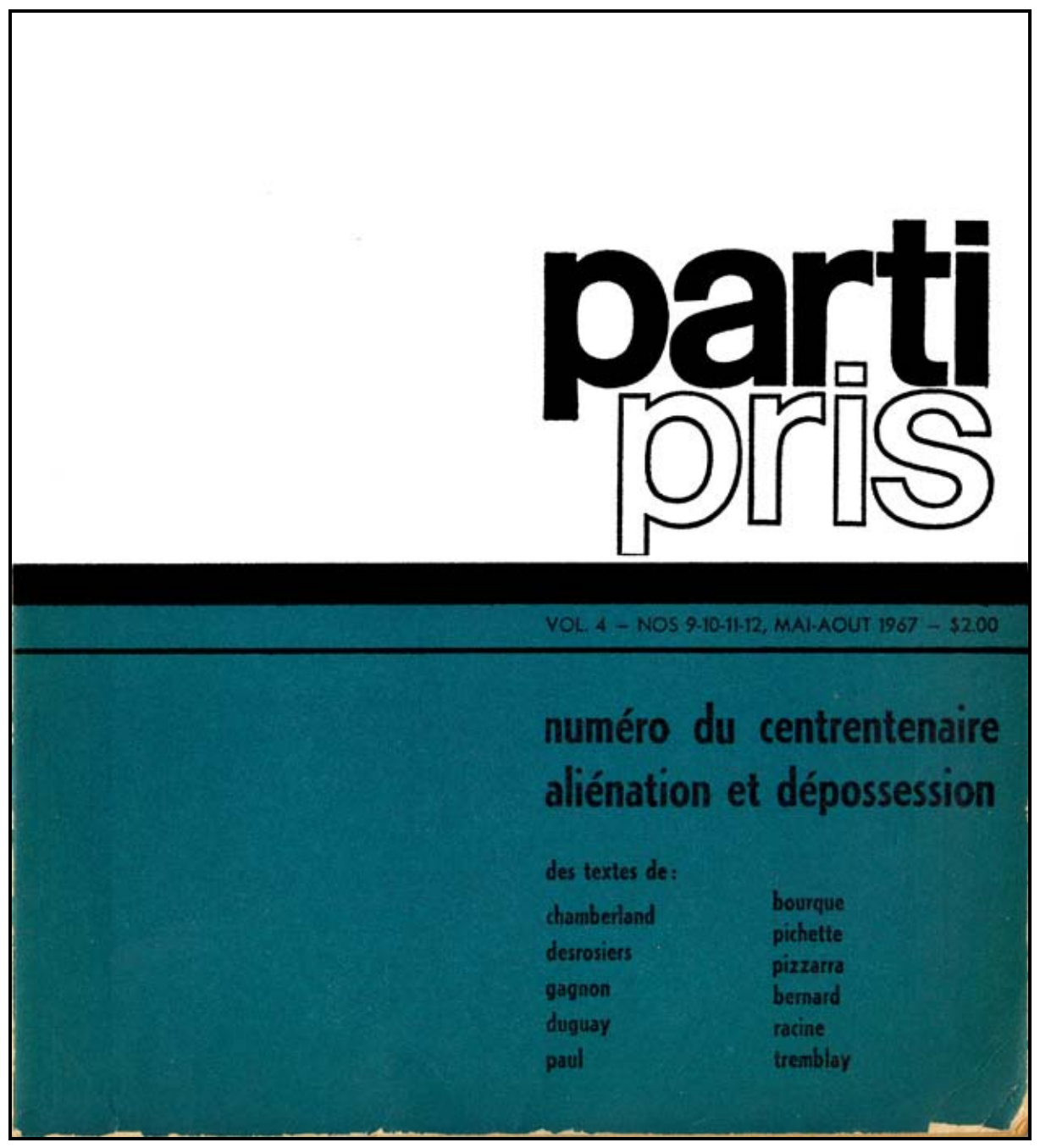

Un article publié dans la revue Parti pris, revue politique et culturelle, vol. 4, nos 9-12, mai-août 1967, pp. 165-181. 


\section{Table des matières}

1. Introduction

2. de l'interdépendance à la dépendance

3. l'image de l'indien : mythe et réalité

4. les comportements sociaux, économiques et politiques

Conclusion : dépossession et prolétarisation 
[165]

\section{Camil GUY \\ Anthropologue québécois \\ "Les indiens du Québec : désagrégation culturelle et prolétarisation.”}

Un article publié dans la revue Parti pris, revue politique et culturelle, vol. 4, nos 9-12, mai-août 1967, pp. 165-181.

\section{Introduction}

Retour à la table des matières

Il semble que les relations entre groupes de cultures différentes tendent toujours à s'organiser en structure de subordination lorsque l'un des acteurs en présence constitue une minorité. Ce modèle assez général des rapports de groupes, qui fait apparaître l'un d'entre eux comme une sous-culture au sein d'une société plus large, ne rend compte que partiellement de la position sociale de l'Indien au Québec. Il ressort avec de plus en plus d'évidence que les pratiques discriminatoires vis-à-vis de ce dernier reçoivent une confirmation légale dans le statut politique qu'on lui reconnaît. Du point de vue québécois, l'Indien représente une personne marginale puisqu'il vit sous la tutelle du gouvernement central. Déjà en retrait de la société par son statut de groupe ethnique minoritaire, il en est une fois de plus coupé par le grillage administratif des Affaires Indiennes. Le pouvoir des chefs et des conseils de bande est sérieusement limité par les prérogatives et les multiples droits de regard des agents administratifs ${ }^{1}$. Ce statut lé-

1 Ce point de vue est abondamment illustré par R.W. Dunning dans "Some aspects of governmental Indien policy and administration", Anthropologica, N.S. vol. IV, no. 2, pp. 209-229. On y voit que cane limitation des pouvoirs d'autrui a été dictée par une politique essentiellement paternaliste. 
gal marginal constitue le deuxième aspect des rapports entre Blancs et Indiens. Le premier système de différences objectives qui s'exprime en termes de race, de coutumes et de traditions étrangères à la majorité et qui servait déjà de prétexte à l'empiètement ou à l'indifférence, se trouve maintenu et renforcé par la constitution des groupes indiens en "bandes" et en "réserves".

Le troisième aspect des relations entre Blancs et Indiens, l'assimilation progressive d'une ethnie à une classe sociale, se comprend par référence aux structures socio-économiques de la société [166] envahissante. Dans la frange sud du Québec laurentien 2, Abitibi, Gatineau, Mauricie, Lac Saint-Jean et Côte Nord, nous avons affaire à une société frontière. Les seuls mobiles de la pénétration blanche, dans le Haut Saint-Maurice par exemple, ont été et restent l'exploitation effrénée des richesses naturelles, surtout forestières et hydrauliques, par l'intermédiaire de grandes et puissantes compagnies. Ces entreprises entraînent avec elles un ensemble de petits commerçants qui en forment plus ou moins la clientèle. Puis vient la classe laborieuse qui suit forcément la source de ses revenus. En règle générale on a préféré les sociétés d'hommes qui constituent les camps de bûcherons à la forme du village forestier. Cette société frontière, on le voit, ne reproduit pas avec exactitude les grandes lignes de celle d'où elle émane ; confrontée à des situations nouvelles, elle les transforme et en accentue certains traits, ceux de la classe laborieuse ici qui paraissent caricaturaux à beaucoup d'égards.

Les structures de ce milieu social et économique et les conditions objectives des contacts ont entraîné l'intégration de l'Indien par le bas, la position que leur réservait logiquement le système. De plus en plus,

2 Les 25,000 Indiens du Québec ne ce ressemblent pas entièrement. On pourrait distinguer en gros trois grandes zones. La première comprendrait les Iroquois de Caughnawaga, les Hurons de l'Ancienne Lorette et d'autres groupes depuis longtemps établis dans la zone d'influence des grands centres urbains. Ces groupes ne nous concernent pas ici. Il y a ensuite les bandes du NouveauQuébec, Baie James, Baie d'Hudson, etc. dont l'éloignement géographique les a préservées jusqu'à maintenant d'une acculturation vraiment Intensive. Nous nous intéressons aux groupes situés entre ces deux extrêmes, dans la forêt boréale exploitée de façon de plus en plus extensive pour ses richesses naturelles de toutes sortes. Rappelons que le nombre total des Indiens au Québec est passé de 16,000 en 1949 à 23,000 en 1964, une augmentation surprenante de plus de $40 \%$ en 15 ans. 
il s'identifie à la classe laborieuse de la société québécoise et adopte un comportement de classe ${ }^{3}$. Occupations saisonnières, revenus limités, dépendance démesurée des allocations sociales diverses, logement précaire, hygiène presque absente, tous ces traits s'appliquent aussi bien à une classe défavorisée dans une société nantie. Ce nouveau glissement vers un statut de classe était à prévoir ; les groupes indiens suivent l'inclinaison déjà décelée au niveau culturel et au niveau politique.

Somme toute la situation se résume à ceci : les groupes indiens actuels de la frange sud du Québec et en particulier la bande de Weymontaching dont il sera question ci-après, présentent à la fois les caractéristiques d'une sous-culture défavorisée, d'une personne légale marginale et d'une classe à la limite inférieure à l'échelle sociale. Ces statuts expliquent une grande part de leur conduite. Ils ont abandonné leur mode traditionnel de subsistance en échange d'une intégra, tien problématique à la société québécoise. Nous allons voir en gros comment s'est posé et comment se pose aujourd'hui le problème de l'intégration pour les Indiens de cette zone en insistant davantage sur l'aspect culturel. Nos observations portent sur un groupe de la Mauricie, la bande de Weymontaching dont la réserve est située près du village blanc ${ }^{4}$ de Sanmaur. Le groupe comprend quatre cents individus répartis dans différents établissements selon le déroulement du cycle annuel des activités.

3 R.W. Dunning adopte cette perspective pour décrire la situation des Ojibwa de l'Ontario, "Some problems of reserve Indien communities : a case study", Anthropologica, N.S. vol. VI, no 1, pp. 3-38.

4 Cette désignation "le Blanc" représente l'inverse de notre terme "L'Indien" grâce auquel nous classifions dans le même groupe des bandes qui sont peu ou pas apparentées, elle comprend les Canadiens français, les Canadiens anglais, les Anglais, les Américains et d'autres. Inutile de dire que dans les deux cas le réflexe socio-culturel est plus rapide que la réflexion scientifique. 


\section{2. de l'interdépendance à la dépendance}

Retour à la table des matières

L'époque récente a marqué essentiellement le passage de l'interdépendance de la bande indienne et d'un secteur avancé de la société euro-canadienne à la dépendance de la première vis-à-vis de la deuxième.

Avant 1940, les Indiens de Weymontaching menaient une existence parallèle, en marge de la société blanche. Un rythme bien défini marque alors la vie sociale du groupe. D'octobre à mai, les familles séjournent en forêt par petits groupes de deux, trois ou quatre. Parfois les familles regagnent la réserve de Weymontaching à l'occasion de Noël et du Nouvel An. De juin à septembre, toute la bande se réunit à nouveau au même endroit. Le seul lien organique qui unit la bande à la société blanche est le poste de traite. Le trappeur échange ses fourrures pour des articles indispensables comme l'équipement de chasse, des vêtements et des denrées alimentaires de base. Ces échanges s'effectuent d'abord avec la compagnie de la baie d'Hudson jusqu'aux environs de 1925, puis avec un traiteur privé. Mariages et baptêmes ont lieu en été à l'occasion de la visite du missionnaire qui ne manque jamais un rendez-vous depuis le milieu du siècle passé. Il a réussi à implanter la religion chrétienne parmi ces bandes. Il ne vient qu'en été et l'hiver l'Indien retrouve parfois ses pratiques "païennes" 5 . Les enfants fréquentent depuis 1925 une école d'été. En hiver ils suivent leurs parents en forêt alors que ceux-ci demandent au commerçant une avance sous forme d'équipement de trappe et de denrées alimentaires en prévision du long isolement qui les attend. L'Indien continue à assurer la majeure partie de sa subsistance sous forme de gibier et de poisson. En dehors de ces liens commerciaux, religieux et scolaires temporaires son existence est libre de toute autre attache à la société blanche. Les trois premières décennies de ce siècle représentent une période relativement prospère pour les Indiens. Le marché des fourrures leur est favorable. Ils construisent autour de l'église catholique et avec leurs propres moyens des maisons sur la réserve de 7,400 acres qu'on leur a cédée en 1851. On voit encore ce village

5 Voir à ce sujet l'essai de J. Rousseau et M. Rousseau "Le dualisme religieux des peuples de la forêt boréale" dans le rapport du 29ième congrès International des américanistes, vol. 2, pp. 118-126, 1952. 
aujourd'hui bien que les maisons se soient passablement [168] détériorées puisqu'on n'y habite plus sauf pendant les quelques jours que dure la visite du missionnaire en été.

Le chemin de fer transcontinental qui atteint Sanmaur en 1913 va accélérer le développe, ment régional. Les chantiers forestiers atteignent bientôt Sanmaur. La main-d'oeuvre est d'abord formée exclusivement de Québécois de langue française. Au cours de la dernière guerre mondiale, plusieurs Indiens font leur entrée dans le monde du salariat. La pénurie de main-d'oeuvre vers 1942 pour la coupe du bois et la drave oblige les compagnies à engager une main-d'oeuvre autochtone. Les Indiens eux-mêmes sont confiants d'y trouver leur profit pour les raisons que nous évoquerons plus bas. Le changement ne s'effectue pas de façon brusque puisque encore aujourd'hui nombre d'Indiens continuent à trapper de façon saisonnière sur leurs territoires de chasse familiaux. Mais dès ce moment, ils font leur entrée définitive dans la société blanche. Concurremment viendront s'ajouter d'autres emplois comme le guidage pour la chasse et la pêche touristiques et la cueillette commerciale des bleuets.

À partir de ce moment, il faut cesser d'envisager le groupe comme une culture distincte et une société marginale possédant sa propre organisation et ses propres sanctions pour lui substituer la constatation plus juste d'une sous-culture et d'un segment de la société blanche à laquelle il appartient d'ores et déjà. L'intervention décisive de l'administration fédérale à compter de ce moment et son importance grandissante dans les années qui suivront témoignent de cette transformation. Ce passage exigera une nouvelle systématisation des rapports sociaux et des rapports ethniques. Au régime de séparation et d'interdépendance va se substituer un régime de dépendance.

Quels sont les facteurs qui ont amené les Indiens à faire ce pas décisif ? Ils y ont été conduits par l'ensemble des conditions qui ont présidé au développement de la Mauricie. Nous nous limiterons ici à l'énumération de ces facteurs qui nécessiteraient autrement un développement trop long pour le cadre de cet article. Nous ne sousestimons pas cet aspect de la question, au contraire, car les attitudes de l'Indien, essentiellement le retrait et la dépendance, se fondent sur son interprétation des conditions historiques qui l'ont amené à être ce qu'il est, i.e. la spoliation de ses droits par les Blancs d'où découle une exigence parallèle de compensation manifeste dans chacun de ses comportements. Ce point de vue s'oppose bien entendu à celui des Blancs 
qui veut que les Indiens refusent obstinément et à leur [169] détriment les bienfaits de la civilisation ; ils ignorent alors le phénomène d'aliénation dont les racines profondes atteignent la dignité même de ceux qu'elle frappe.

Le premier de ces facteurs de passage de l'interdépendance à la dépendance réside dans l'usurpation du territoire indien par les trappeurs blancs d'abord, par les compagnies forestières et les clubs ensuite. Davidson ${ }^{6}$ qui écrit en 1928 nous rappelle que la bande de Weymontaching comprend des individus autrefois membres d'une autre bande, celle de Coucoucache, qui s'est désintégrée vers 1910 par suite de l'invasion par les blancs de leur territoire. Il n'était, dit-il, plus possible de vivre à la façon traditionnelle. La bande de Weymontaching elle-même ne fut pas exempte de telles intrusions. Le journal des chefs formule des plaintes à cet égard dès les années 1920. Plusieurs sections de l'ancien territoire de la bande ont été allouées à des trappeurs blancs, la plupart du temps des gardiens de clubs privés de chasse et de pêche. Cette allocation du territoire à des clubs privés blancs apparaît comme une autre forme d'invasion ; les Indiens devaient parfois se cacher pour chasser et pêcher sur ces territoires même si leur droit de trapper des animaux à fourrures était reconnu. Il y eut maintes escarmouches entre Indiens et Blancs allant jusqu'à l'incendie des camps et de l'équipement des premiers. Les droits de chasse des Indiens ont depuis été reconnus à peu près partout sur leur territoire sauf sur quelques enclaves, mais à une époque où ils commencèrent à s'en désintéresser par la force des choses. Voici un extrait du discours d'un ancien chef de Weymontaching prononcé en 1939 devant l'évêque des Trois, Rivières et dans lequel il exprime les désirs de son peuple : "Nous aimions beaucoup l'autre évêque ; aujourd'hui, on nous demande de t'aimer comme l'autre. On t'aimerait davantage si tu disais à Duplessis, le roi du Québec : "Défends aux Blancs de faire la chasse aux animaux à fourrures. Eux, les Blancs, ont de l'ouvrage parmi eux pour gagner leur vie. Nous, notre vie, c'est la chasse et la pêche." Et après avoir rappelé que la compagnie de la baie d'Hudson avait fermé son bureau au Weymontaching parce que les animaux étaient trop rares, le chef concluait : "Si on veut nous faire mourir, qu'on laisse les Blancs faire la chasse avec leurs [170] beaux fusils et leurs beaux canots." 7

6 "Notes on the Têtes de Boule ethnology", Amarican Anthropologist, vol. 30, pp. 28-29.

7 cité dans Y. Thériault, "L'apostolat missionnaire en Mauricie", collection "L'histoire régionale". No 7. éditions du Bien Public, Trois-Rivières, 1951, pp. 112-113. 
Le déboisement du territoire par les compagnies forestières se présente comme une nouvelle forme d'usurpation du point de vue de l'Indien et comme un facteur important de bouleversement de l'équilibre écologique ancien sur lequel était basé leur mode de vie. Les transformations écologiques qu'entraînent ces vastes opérations sont évidentes ; la forêt disparaît presque complètement. Récemment on a expérimenté une nouvelle technique qui consiste à faire passer le feu derrière les machines et les bûcherons. Cette fois il n'y a plus de doute possible, la destruction a été menée à terme pour une période de soixante-dix ou quatre-vingts ans. Avant ces techniques perfectionnées, les conséquences des coupes sélectives n'étaient pas moins immédiates ; les chaussées de castor sautent à la dynamite parce que les eaux inondent les routes de charroyage du bois; les coupes détruisent les portages tracés depuis des générations par l'Indien pour se déplacer sur son territoire de chasse.

À ces différents facteurs, il faut ajouter les fluctuations du marché des fourrures, la difficulté de survie en forêt et un changement d'attitude chez l'Indien qui au péril de la vie en forêt préfère se rapprocher un peu des blancs et rendre ainsi moins aléatoire la question de sa subsistance.

Les détails de cette évolution importent moins que les conséquences de cette nouvelle orientation sur le mode de vie de l'Indien. En conséquence il importe plus de restituer dans ses grandes lignes le point de vue de l'Indien que d'insister sur les modalités de cette évolution. Notons seulement ce que l'Indien lui-même en a retenu. Dans ce cheminement historique de plusieurs décennies menant de l'interdépendance à la dépendance, il a été lésé de ses droits sur tous les plans. Car l'abandon de son mode traditionnel de subsistance signifiait également une déculturation progressive, la culture des peuples chasseurs et pêcheurs étant liée de très près aux activités de subsistance. La trappe commerciale permettait de perpétuer quand même ce mode de vie ; la coupe du bois ne le permet plus. Il a été dépossédé, il a perdu sur tous les points. Sa réaction en est une d'abdication et de retrait pur et simple. Ayant perdu son identité ancienne, il refuse également de s'identifier à la classe laborieuse québécoise vers laquelle sa nouvelle situation le fait glisser. Il a été vaincu et cette situation a développé chez lui un sentiment très réel d'infériorité. 


\section{[171]}

Si l'on regarde en arrière, on voit que les relations entre Blancs et Indiens se répartissent en un certain nombre de catégories possédant un dénominateur commun ; elles sont en effet déductibles à cette seule relation d'inférieur à supérieur. Le missionnaire et le médecin sont plus forts que le shaman; le chasseur dépend largement des avances que le traiteur veut bien lui consentir ; l'autorité du surintendant des Affaires Indiennes surpasse celle du chef ; il en va de même pour l'instituteur vis-à-vis des parents indiens ; la RCMP est plus forte que tout Indien; les compagnies forestières font fi des droits séculaires des Indiens; (plus récemment) le bûcheron québécois surclasse son homologue indien et le trappeur blanc paraît également vis-à-vis du pourvoyeur. On pourrait illustrer facilement chacune de ces relations et les réduire au rapport simple évoqué plus haut. Voyons plutôt comment l'Indien réagit devant deux systèmes culturels, deux systèmes de valeurs, le sien propre qu'on dévalorise et celui de la société blanche qu'on lui présente comme un mieux-être et comme le substitut logique du premier système ; cette analyse doit tenir compte de l'attitude des blancs vis-à-vis des Indiens, attitude qui influence souvent le comportement de ces derniers.

\section{3. l'image de l'indien : mythe et réalité}

\section{$\underline{\text { Retour à la table des matières }}$}

Cet ensemble de relations à caractère systématique s'appuie sur les nombreux traits stéréotypés dont le Blanc affuble l'Indien et qui marquent la supériorité du premier en prêtant au second des conduites condamnables et préjudiciables à ses propres intérêts. L'un de ces stéréotypes dit que l'Indien est malpropre; ses campements toujours temporaires ne comportent pas d'accommodation hygiénique et les déchets s'éparpillent autour des tentes ; cette accusation atteint même sa personne. Un autre veut que ce soit un soûlard et un trouble-fête.

C'est aussi, croit-on, un paresseux sans ambition aucune, qui n'a pas d'égard pour son propre bien-être. On pourrait continuer l'énumé- 
ration ${ }^{8}$. Plusieurs histoires courent au sujet de sa moralité ou de son immoralité. L'expression populaire de "sauvage" les résume tous avec tout ce que cela implique de déshonorant, de non-civilisé, de "naturel". Ces qualificatifs suffisent à les déclasser définitivement sans qu'on ait cherché à expliquer leur origine et les fonctions qu'ils remplissent dans certains cas. Ainsi les conditions de vie de l'Indien et l'absence fréquente [172] de motivation donnent un fonde, ment de vérité à ces stéréotypes, mais sont-ils causes ou résultats ? De plus on peut voir dans les conduites de l'Indien en état d'ébriété, agression, pleurs soudains apparemment sans cause, un mécanisme social utile permettant de libérer une certaine dose d'anxiété qui a une autre origine ; on retrouve ce phénomène chez certains Québécois d'ailleurs.

Les Indiens ont eux-mêmes inventé un mythe pour échapper à cet encerclement. En réponse à ces accusations de la société euro-canadienne, on a vu se dessiner un mouvement nativiste dont Jules Sioui de l'Ancienne Lorette s'est fait au Québec le porte-parole et le promoteur. Cet appel a reçu une certaine audience auprès des Indiens de Weymontaching et d'ailleurs. L'Indien y reprend le même point de vue, mais il inverse les termes de la relation. Ce mouvement veut en effet montrer la pureté de l'Indien qui isolé providentiellement en Amérique et protégé à dessein par Dieu, n'a pas été touché par la faute d'Adam et d'Ève. Revendication idéologique par les croyances et politique parles diverses pressions exercées à Ottawa à partir de cette position de force, notamment contre la conscription en 1942. Une étude d'ensemble de ce phénomène révélerait sans doute des faits utiles à l'interprétation de l'histoire indienne. Aujourd'hui ce mouvement n'a plus de prise chez les éléments avancés de la communauté indienne qui dépouillent leurs revendications de cet aspect religieux pour se limiter à l'aspect socio-politique.

Il existe une deuxième image largement mythique, romantique, idyllique même que véhicule l'inconscient collectif des Blancs. En, gros, c'est celle de l'individu qui vit dans l'intimité de la nature. On envie cette camaraderie de tous les jours qui semble receler bien des

8 Ces stéréotypes collent de si près à la situation indienne qu'on retrouve exactement la même énumération dans l'étude de E.J. James qui porte sur les Ojibwa du Wisconsin, "Socio-psychological dimensions of Ojibwa acculturation", American Anthropologist, vol. 63, no. 4. 1961, pp. 721-746. Nous nous inspirons de cette étude dans les pages qui suivent. 
secrets et fournir bien des satisfactions. On y voit une certaine image de la liberté, à l'état sauvage pour ainsi dire, où l'individu plutôt que d'obéir à des horaires et se soumettre à un métier, va ou bon lui semble, fait un peu ce qu'il lui plaît. C'est d'ordinaire à ce dernier individu que s'adressent par exemple les chasseurs blancs à la recherche d'un guide indien qui leur enseignera les choses de la nature ; c'est aussi celui que chantent nos chansonniers. Pour eux, l'Indien exerce une forme de médiation entre la nature et la culture, entre la forêt et la civilisation urbaine et on se demande si cette hypothèse ne pourrait être élargie pour s'appliquer à toute la collectivité blanche. Inversement, le Blanc en sa qualité de pour, [173] voyeur représente à bien des égards pour l'Indien un substitut à son univers religieux, aux forces religieuses dont la manipulation assurait les succès de la chasse.

Les conditions réelles de la vie de l'Indien aujourd'hui témoignent de l'écart immense qui sépare ces deux représentations, l'une qui se fait à coups de stéréotypes et l'autre qui reflète une certaine conception de l'homme libre dans la nature ; entre les deux il y a un large espace ou s'inscrira le vrai visage de l'Indien. Mais ses réactions se fondent largement sur ces deux images que lui renvoient de lui-même les Blancs et qu'il intériorise sous des formes diverses. Dans sa tentative de soutenir la comparaison avec le Blanc, il part perdant ou à tout le moins fortement infériorisé. Ses comportements sociaux, économiques et politiques, souvent incompris de ceux qui le côtoient, reflètent à beaucoup d'égards les attitudes de la société euro-canadienne. S'il adopte un comportement contraire à celui qu'on lui reproche, il trouve une voie d'accès à la nouvelle société ; mais, ce faisant, il abandonne ses dernières tranchées, il donne raison à ses détracteurs et adopte les valeurs de l'autre. Écartelé entre sa propre interprétation de l'histoire et celle des Blancs, il hésite et se divise. 


\section{4. les comportements sociaux, économiques et politiques}

$\underline{\text { Retour à la table des matières }}$

De quelle façon les rôles de l'Indien s'accommodent-ils de ces statuts différentiels et de ces représentations collectives qui en forment la répartie dans l'esprit des Blancs ? Le cycle annuel des activités comprend la coupe du bois, la drave, le guidage pour la chasse et la pêche touristique, la trappe, la cueillette commerciale des bleuets. On remarquera tout de suite que ces activités se répartissent en deux groupes, celles qui se rattachent au mode traditionnel de subsistance, les quatre dernières, et celles qui marquent l'empire de la nouvelle économie, la coupe du bois et la drave. Cette répartition n'est pas arbitraire; elle constitue dans l'esprit des Indiens deux orientations du système culturel, l'une conservatrice et mieux adaptée à leurs traditions, l'autre nouvelle et introduite du dehors. Les motivations varient dans les deux cas. Il est à remarquer que ces activités ne se succèdent pas nécessairement selon un ordre chronologique, mais qu'elles [174] peuvent avoir lieu en même temps et qu'elles supposent donc un choix. La coupe du bois se fait de nos jours en été comme en hiver. Comment s'exerce le choix de l'Indien et comment remplit-il ses rôles?

La coupe du bois et la drave exigent de lui une stabilité au travail à laquelle il n'est pas accoutumé. Les contremaîtres de la compagnie demandent de sa part une ambition et un désir de gain de sorte que son rendement soit maximum ; ils exigent une certaine retenue dans la consommation de l'alcool pour qu'il puisse se présenter régulièrement à l'ouvrage ; ils demandent qu'il ne quitte pas le travail à la première occasion venue pour une excursion de chasse, de trappe ou de guidage. Seule cette stabilité au travail assure une production constante et maximum. L'Indien qui obéit aux normes de la société traditionnelle possède un tout autre cadre de référence. Il ménage le travail et les ressources naturelles quand les besoins primaires sont satisfaits. Il ne voit pas la nécessité d'accumuler, habitué qu'il est à éviter le gaspillage s'il veut survivre en forêt ; le trappeur ne doit pas décimer la population des castors s'il veut que la colonie se renouvelle et fournisse une récolte annuelle. Les réserves de bois des compagnies sont considérables et leur but premier est de produire vite et beaucoup. C'est l'esprit de conservation opposé à la politique de production maxi- 
mum. Il se peut fort bien que cette attitude soit transférée de la chasse et la trappe à la coupe du bois. Les compagnies forestières représentent en effet le nouveau mode de subsistance. C'est une source de revenu que l'on exploite pour assurer sa subsistance sans nécessairement adopter pour cela les valeurs et les normes de la production capitaliste. Sur ce point toutefois tous les Indiens n'adoptent pas le même comportement. Quelques-uns ont choisi de travailler à la façon des Blancs en essayant d'échapper aux accusations de paresse et de manque d'ambition. Ils ne vont presque plus trapper ; ils s'absentent seulement pour guider les touristes à la chasse à l'orignal et pour encaisser pendant quelques mois les bénéfices de l'assurancechômage. L'un d'entre eux a même acheté une maison dans le village blanc de Sanmaur. Il éprouve toutefois des difficultés du fait que les obligations liées aux statuts parentaux traditionnels qui continuent à jouer l'obligent à héberger beaucoup de parents et à subvenir malgré lui à certains de leurs besoins. Quant aux Blancs, ils se [175] réjouissent de ces initiatives. Un autre groupe se comporte tout à l'opposé de ce dernier. Ils n'ont pratiquement jamais travaillé à la coupe du bois ; ils continuent à pourvoir à leur subsistance à la façon traditionnelle. Ils sont dans l'ensemble plus âgés que ceux du premier groupe. Le reste de la bande va d'une activité à l'autre selon le déroulement du cycle annuel. "Ce sont des nomades", disent les Blancs, d'un air de dire : "On ne les corrigera jamais." Quand arrive la saison de la chasse, la plupart des Indiens partent guider. Cela dure près de trois semaines. Ensuite plusieurs vont trapper en forêt par périodes d'une, deux ou trois semaines, en automne et au printemps. Au mois d'août nombre de familles dressent leur tente au milieu des pousses de bleuets.

Les motivations à un travail stable et régulier supposent l'assimilation des valeurs qui s'y rattachent. Ce n'est pas chose faite comme on le voit sauf pour quelques exceptions qui souffrent elles-mêmes de l'emprise des obligations familiales traditionnelles, un mécanisme du système visant peut-être à sa propre défense. James ${ }^{9}$ fait remarquer que le guidage, mis à part les sollicitations financières, est une autre façon de se faire accepter par les Blancs et non pas seulement un retour romantique à la vie en forêt. Il appuie son hypothèse sur le fait que les Blancs valorisent chez l'Indien sa connaissance de la nature, son intimité avec le milieu naturel. La valeur de l'Indien s'en trouve rehaussée à ses propres yeux. Il se plaît dans cette activité où il est bien accepté par les Blancs. Il s'y adonne d'autant plus volontiers qu'il est d'ordinaire bien rémunéré par le chasseur heureux. La chasse et la trappe commerciale correspondent, elles, à un retrait de la situation

9 Op. cit., p. 729. 
qui lui est faite dans la société blanche, dans les chantiers de bûchage 10 , à une orientation plus conservatrice de la culture. Ce fait est confirmé par la constatation que malgré de multiples séjours en forêt, l'Indien rapporte peu de fourrures, du moins jamais en quantité suffisante pour être comparé favorablement aux revenus qu'il peut tirer de la coupe du bois. Ses motivations ne sont donc pas d'abord d'ordre économique ; il opère un retour plutôt à l'ancien système culturel. En forêt il a souvent d'ailleurs un régime d'alimentation qu'il trouve plus convenable, constitué surtout de gibier et de poisson ; l'approvisionnement dans les camps de bûchage est parfois difficile et les moyens de conservation sont toujours précaires. De la même façon, la cueillette commerciale des bleuets répond à un même besoin de retourner à un monde qui n'est pas, du moins en apparence, dominé directement par les Blancs.

\section{[176]}

Ceux-ci voudraient également voir les Indiens remplir d'autres rôles, décider de leur avenir dans un sens comparable à celui du citoyen normal dans la société. Ainsi on les blâme de continuer à vivre sous la tente ; ceci apparaît à la fois comme une accusation et comme un défi. L'hygiène et les services publics de toutes sortes ne peuvent être pensés en dehors du type d'habitation et d'établissement du Blanc. Il faut dire que malgré leur intégration partielle à l'économie nouvelle, la majorité d'entre eux habitent encore sous la tente ce qui s'accorde bien avec leur mobilité occupationnelle et géographique, mais bien malgré eux comme nous allons le voir.

Plusieurs familles possédaient déjà une maison sur la réserve de Weymontaching dont la construction remontait à vingt ans ou plus. Ils y vivaient de juin à septembre. La suspension des cours d'été due à la construction d'un pensionnat en dehors de la Mauricie, a fait disparaître la dernière raison qui maintenait le groupe sur la réserve en été ; la compagnie de la baie d'Hudson avait déjà quitté vers 1925. Les familles sont dispersées sur le territoire de la bande selon la location de leur territoire de chasse et selon la location des sources de revenus du moment, habituellement par petits groupes d'une dizaine de familles. Cette situation a subsisté jus-

10 Lorsqu'il soupçonne par exemple à tort ou à raison que les compagnies forestières accordent aux Québécois, bûcherons plus efficaces, les meilleures coupes et leur réservent le petit bois et les brûlés malpropres. 
qu'à maintenant. Les Indiens ne reviennent sur la réserve qu'une semaine en été pour accueillir le missionnaire. La réserve est d'ailleurs difficilement habitable en hiver et au printemps par suite de la construction des barrages sur le Saint-Maurice ; le niveau des eaux varie à intervalles irréguliers, ce qui fait qu'on ne peut plus compter sur le pont de glace pour aller se ravitailler à Sanmaur sur l'autre rive. Certains d'entre eux ont construit ailleurs dans ce territoire de petites cabanes, habituellement dix pieds par douze, en bois rond ou en planches recueillies dans des camps abandonnés de bûcherons. Mais leur mobilité occupationnelle et géographique font qu'ils n'y habitent que rarement. Le plus souvent la plupart des familles sont sous la tente, en hiver comme en été. Les Affaires Indiennes aimeraient que les Indiens choisissent un emplacement pour la construction d'un village dont le coût serait partagé entre le gouvernement, la bande dont le fond est assez impressionnant (environ \$150,000 provenant de la vente du bois sur la réserve) et les familles concernées. Les Indiens désirent également avoir une maison. [177] On croirait que l'endroit est déjà trouvé : la réserve de Weymontaching puisqu'il y a déjà un embryon d'organisation communautaire.

C'est bien là le point de vue des Indiens pour qui la réserve prend figure de symbole un peu comme dans le cas des activités économiques qui les rattachent au système culturel traditionnel. L'usurpation de leur territoire sous des formes diverses n'a laissé intacte que cette bande de terrain le long du Saint-Maurice à l'embouchure de la rivière Manouane que le gouvernement leur a cédée il y a un siècle. "Ce terrain nous appartient. Nous sommes ici chez nous." Pas un Blanc cette fois ne peut le contester puisque son propre système légal reconnaît le droit de propriété des Indiens. En retrait des routes de la Mauricie, pas un Blanc ne pénètre sur la réserve sauf sur invitation de la bande à l'occasion de la célébration solennelle de la Fête-Dieu au cours de laquelle l'Indien montre aux Blancs un visage pour eux acceptable puisqu'il fête avec grands apparats un événement qui leur tient également à coeur.

Une procession défile dans le village sur un tracé bordé par de jeunes bouleaux coupés la veille et plantés en deux rangées. Un orchestre comprenant guitare, tambour et violon forme la tête du défilé (cette pratique a été abandonnée depuis quelques années), puis vient le groupe des femmes, ensuite le Saint-Sacrement sous le dais et finalement le groupe des hommes qui ferment la marche. Toute la communauté s'ébranle dans cet ordre et se rend au reposoir où les choeurs dirigés par un vieil Indien accompa- 
gnent le salut du Saint-Sacrement. Deux fillettes en robe blanche et portant des ailes de carton dur s'agenouillent de chaque côté du reposoir et leurs mains jointes invitent les fidèles au recueillement. Il y a toujours quelques visiteurs pour participer à cette cérémonie ; les Indiens vont les chercher en canot sur l'autre rive du Saint-Maurice à proximité de Sanmaur. Il ne s'agit pas de pratiques "païennes", mais de célébrations hautement "civilisées" avec toutes les grandes pompes qu'exige un tel rappel religieux. En ceci l'Indien surpasse même le Blanc; il le sait et il invite ce dernier à en être témoin. Le Blanc réagit avec beaucoup de ferveur et se réjouit de le trouver ainsi. C'est une façon pour l'Indien de se faire accepter tout en témoignant d'une dévotion et d'une sincérité réelle dans ce qu'il fait.

Fermons cette parenthèse et revenons à la valeur symbolique de la réserve. C'est pour l'Indien un espace inviolé par les Blancs et par là plus ou [178] moins sacré. Il peut s'y réfugier en toute confiance, sans contestation possible de la part de qui que ce soit. Toucher à la réserve, c'est comme une atteinte à ce qu'il leur reste de fierté et d'honneur, c'est menacer leur dernier refuge. Et pourtant cette menace plane sur le groupe depuis près de vingt ans alors qu'une compagnie hydroélectrique décidait de construire un barrage de retenue des eaux en aval de Weymontaching. L'exécution de ce barrage doit causer l'immersion d'une grande partie de la réserve dont le vieux village indien et la rendre inhabitable. Ce projet n'a pas encore été réalisé, mais il tient toujours et empêche la construction de nouvelles maisons comme il a empêché l'entretien et la rénovation des maisons déjà érigées qui ont été depuis lors laissées à l'abandon.

Devant cet état de choses, un ancien officier des Affaires Indiennes leur a proposé de se fondre aux bandes de Manouane et d'Obedjiwan occupant le voisinage immédiat, ce qui était impensable du point de vue des Indiens de Weymontaching d'abord soucieux de préserver leur autonomie de groupe. On en est ensuite venu à des suggestions apparemment plus réalistes. Un projet d'achat de terrain à proximité d'un village québécois de la Mauricie, qui avait obtenu l'assentiment des Indiens en désespoir de cause, a été rejeté par l'hostilité des villageois à la venue des Indiens sur leurs limites. C'était un affront dur à encaisser. Un autre projet sous forme d'échange d'une partie de la réserve pour un terrain de la couronne a lui aussi été repoussé, par les Indiens cette fois jugeant les conditions du marché inacceptables telles qu'elles étaient formulées par la compagnie détentrice dès droits de coupe de bois à cet endroit. Tous les projets ont jusqu'ici avorté de semblable façon. Les modalités de ces projets et de ces rejets 
importent moins que le fait de l'existence même de ce problème alors qu'il y a quelques années personne ne leur aurait disputé ce territoire. Le groupe reste avec un fond de bande de quelque $\$ 150,000$ qu'il désire d'ailleurs affecter non pas à l'achat d'un terrain mais à la mise sur pieds d'éventuels services communautaires, et dans l'impossibilité de vaincre les intérêts des uns et des autres dans la Mauricie. La réaction plus cou moins consciente des Indiens s'apparente encore une fois au sentiment de dépossession. L'histoire se continue et l'interprétation qu'ils en donnent se confirme une fois de plus. À cela [179] s'ajoute la difficulté d'arriver à une entente parmi les Indiens concernés, peu habitués de prendre des décisions intéressant tout le groupe. Chaque petit groupe pousse de l'avant son propre projet ou trouve quelque défaut à ceux des autres. Seule la réserve réussit à faire tout de suite l'unanimité. Cette difficulté découle notamment de l'influence limitée de la chefferie qui est supplantée par les pouvoirs étendus des Affaires Indiennes.

Devant toute cette affaire, la réaction des Indiens est une fois de plus le retrait. Ils sentent leur impuissance d'autant plus vivement que leur pensée est démunie de moyens d'action devant toutes les "technicalités" qu'impliquent l'administration, les droits des compagnies, des gouvernements fédéral et provincial, etc. Les informations fournies par les responsables n'atteignent pas toujours les intéressés ou alors elles subissent en cours de route des déformations qui les rendent inutilisables. Les responsables doivent s'occuper d'administration et de trois ou quatre bandes et ils n'ont le temps de faire qu'une brève visite de temps à autre. L'Indien se détourne et s'accroche à ses droits acquis, i.e. la réserve même si elle est menacée d'immersion.

Cette situation concourt à maintenir le bas niveau de développement de cette bande. La dispersion des familles justifie aux yeux du ministère de la santé l'absence de dispensaire et d'infirmière dans la région. Les conditions hygiéniques se maintiennent à un niveau très bas et maints incidents fâcheux souvent épidémiques ne peu, vent être évités. Pour les mêmes rai, sons, il n'y a pas d'école locale comme c'est la coutume pour les enfants du cours primaire. Ceux-ci entrent au pensionnat de Pointe Bleue au Lac Saint-Jean dès l'âge de cinq ou six ans, ce qui n'est pas pour réjouir les parents qui perdent leur autorité dans l'éducation de leurs enfants. Ceux-ci par contre passent tous les ans d'un système culturel où la socialisation de l'enfant se fait selon des normes permissives et pratiques à un autre où une discipline stricte et une dépendance rigide en termes de satisfaction des besoins ré- 
gissent le passage de l'enfant à l'adulte. En somme tout ce qu'il faut pour poser à l'adolescent un problème d'identité difficile à résoudre. Le processus de déculturation mis en branle par l'abandon des activités économiques de chasse, de trappe et de pêche, qui constituaient le fondement de la culture traditionnelle, se poursuit ici avec encore plus d'intensité.

[180]

\section{conclusion dépossession et prolétarisation}

Retour à la table des matières

Les multiples dimensions de ce qu'on est convenu d'appeler le problème indien et dont nous avons essayé de dégager ici certains aspects, établissent clairement qu'on est loin de l'époque où un choix idéologique s'offrait entre l'intégration, l'assimilation, le régime de séparation ou d'autres formules du genre. La réflexion a été sur ce point dépassée par les événements et on peut dire que l'intégration était un fait accompli dès que la société euro-canadienne prenait possession de ces forêts boréales. Cette intégration, on l'a vu, n'allait pas de soi, mais posait à son tour des problèmes multiples. Le débat doit donc se déplacer au niveau des modalités de cette intégration à la société et à l'économie canadienne. Or cette analyse a montré que l'intégration au moment où elle se faisait, définissait du même coup ses modalités en faisant glisser, par une espèce de logique interne, le groupe indien dans la classe laborieuse. Le schéma plus simple des rapports de groupes à culture différente acquiert de ce fait une nouvelle dimension, celle qui ressort de l'analyse des classes et des types de production et nous revenons ainsi à notre propos initial. Vu dans son ensemble, ce processus apparaît finalement assez simple ; l'usurpation du territoire en marque le début, la transformation du chasseur et du trappeur en bûcheron ou main-d'oeuvre nécessaire aux compagnies d'exploitation forestière s'inscrit dans son prolongement. Nous sommes donc au deuxième stade d'un même processus dont le déroulement n'a pas échappé aux Indiens eux-mêmes. Les formes de sa résistance sont restées les mêmes malgré qu'elle n'ait forcément rien de très 
efficace. Perdant progressivement leur identité ancienne, ils hésitent à se définir exclusivement comme bûcherons dans un monde où les valeurs dominantes les attirent et les repoussent tout à la fois. La situation en est là pour le moment à Weymontaching.

\section{Camil Guy}

\section{Fin du texte}

